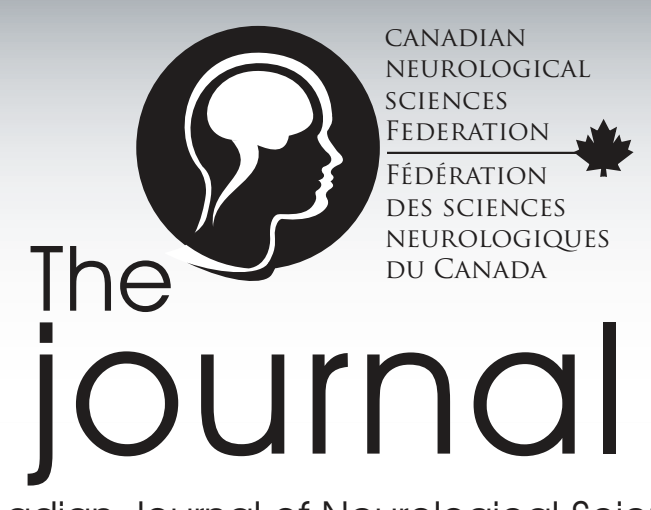

Canadian Journal of Neurological Sciences

Volume 39 Numéro 6 (supplément 5) Novembre 2012

\title{
4e Conférence canadienne consensuelle sur le diagnostic et le traitement de la démence
}

Publié sous forme de supplément évalué par les pairs au The Canadian Journal of Neurological Sciences 


\title{
4e Conférence canadienne consensuelle sur le diagnostic et le traitement de la démence
}

\author{
S. Gauthier, C. Patterson, H. Chertkow, M. Gordon, N. Herrmann, K. Rockwood, P. Rosa-Neto, \\ J.P. Soucy au nom des participants de la CCCDTD4*.
}

Can J Neurol Sci. 2012;39: Suppl 5: S1-S8

Depuis 1989, trois Conférences canadiennes consensuelles sur le diagnostic et le traitement de la démence (CCCDTD) $)^{1-3}$ ont donné lieu à des recommandations portant sur le diagnostic et le traitement de la maladie d'Alzheimer (MA) et des démences apparentées. Les précédentes CCCDTD ont essayé d'établir des recommandations destinées aux professionnels de la santé de toutes les disciplines traitant des patients atteints de démence, tels que les praticiens de première ligne, les neurologues, les gériatres et les psychiatres. Ces recommandations ont été publiées dans diverses revues médicales afin de rejoindre un grand nombre de personnes (tels les lecteurs du Journal de l'association médicale canadienne (JAMC) ainsi qu'un lectorat plus spécialisé (comme celui du Journal canadien des sciences neurologiques et du journal Alzheimer's \& Dementia). Suite à la dernière CCCDTD organisée en 2006, le JAMC a publié une série d'articles basés sur des histoires de cas accompagnées de recommandations pour chaque stade de la MA (asymptomatique à risque ${ }^{4}$; troubles cognitifs légers ${ }^{5}$; démence légère à modérée ${ }^{6,7}$; démence sévère $^{8}$ ). La 4ème CCCDTD a eu lieu en mai 2012 à Montréal et avait pour objectif premier la mise à jour des anciennes recommandations pour les approches diagnostiques de la $\mathrm{MA}^{9}$ en tenant compte des critères proposés par le Groupe international de travail $(\mathrm{GIT})^{10,11}$ de même que les recommandations énoncées par les groupes de travail de l'Institut national sur le vieillissement et de l'Association Alzheimer (INV/AA) ${ }^{12-14}$ de même que de la perspective canadienne publiée de façon concomitante ${ }^{15}$.

\section{MÉTHODES}

Cette conférence a utilisé la méthode du groupe AGREE, respectant 20 des 23 critères existants. Bien que les précédentes CCCDTD ont eu recours au système de notation axé sur des études développées par le Groupe d'étude canadien sur les soins de santé préventifs, nous avons cette fois-ci essayé de suivre, dans la mesure du possible, le système GRADE tout en respectant les recommandations actuelles sur la conduite des conférences consensuelles ${ }^{17}$.

Des revues de littérature préparées par des groupes de travail ont été publiées sur un site Web protégé par un mot de passe, accessible à tous les participants de la conférence, et où ces derniers étaient invités à faire des commentaires. Des recommandations, qui étaient modifiées le cas échéant suite à ces commentaires, ont ensuite été soumises à un vote électronique. Des organismes fournissant des soins aux personnes atteintes de démence ont été également sollicités afin de nommer des délégués. Ces derniers avaient librement accès aux articles de documentations, ont été encouragés à les commenter et ont eu le droit de voter sur les recommandations. La clôture du vote électronique a été fixée au jour précédent l'assemblée de la conférence, qui s'est déroulée les 4 et 5 mai 2012 à Montréal. Lors de la conférence, chaque point a été brièvement abordé avant de passer au vote concernant chacune des recommandations. Tous les participants (à l'exception des observateurs de l'industrie) ont eu le droit de voter. En cas d'absence de consensus, les votes électroniques des participants à la conférence mais qui n'étaient pas présents à l'assemblée, ont été pris en compte. Comme à chaque CCCDTD, on a considéré que le consensus était obtenu lorsque $80 \%$ ou plus des participants à la conférence avaient voté pour la recommandation. Un consensus partiel représentait un appui de 60 à $79 \%$ des membres admis à voter. Les recommandations faisant l'objet d'un consensus ( $\geq 80 \%$ ) sont énoncées dans les tableaux de cet article. Les recommandations n'ayant obtenu qu'un consensus partiel ou aucun consensus font l'objet de commentaires dans le texte. Le niveau d'évidence est mentionné dans les tableaux lorsque cela est possible. Les recommandations s'appliquant uniquement au domaine de la recherche sont identifiées par un « $\mathrm{R} »$ dans les tableaux.

La majorité des recommandations s'adressent principalement aux spécialistes traitant les patients atteints de démence compte tenu de la nature des sujets abordés : définitions/nouveaux critères de diagnostic de la MA, utilisation de la neuroimagerie et des biomarqueurs liquides, apparition précoce de la démence, démence à progression rapide. Ces recommandations seront été publiées en ligne en novembre 2012 par le Journal Canadien de Gériatrie. Les traitements symptomatiques, qui sont pertinents pour les médecins traitants, sont également discutés dans cet article.

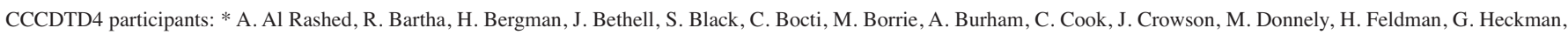
D. Hogan, G.Y.R. Hsiung, G. Inglis, C. Jacova, R. Laforce, K. Lanctot, L. Lee, K. Leclair, M. Masselis, F. Massoud, A. Moore, S. Prasad, K. Rabheru, D. Sadovnick, L. Trudeau, I. Vedel, M. Williams.

ReÇU 17 JUILlET 2012. Révisions FinALES SOUMIS 24 JUILLET 2012.

Correspondance: Serge Gauthier, McGill Center for Studies in Aging, 6825 LaSalle Blvd, Montreal, Quebec, H4H 1R3, Canada. Email: Serge.gauthier@mcgill.ca. 


\section{Définitions/nouveaux critères diagnostiques}

L'évolution de la façon dont la MA peut être diagnostiquée au vu des nouveaux critères mis en place par les États-Unis nous a poussé à réviser les critères canadiens pour le diagnostic de la MA. Le GIT, dirigé par Bruno Dubois et Howard Feldman, a proposé de diagnostiquer la MA bien avant l'apparition des signes évidents de démence, en ayant recours à un phénotype clinique spécifique (troubles de la mémoire de type hippocampique) accompagné de positivité d'un de divers biomarqueurs $^{10}$. En 2011, trois groupes de travail de l'INV/AA ont proposé des critères pour poser un diagnostic de démence provoquée par la $\mathrm{MA}^{12}$, de troubles cognitifs légers causés par la $\mathrm{MA}^{13}$ et de MA asymptomatique ${ }^{14}$. Le groupe sur la phase asymptomatique a très clairement adopté la proposition de Dubois/Feldman pour ce qui est de la dépendance de ce diagnostic sur la présence de biomarqueurs positifs. En outre, les déclarations portant sur les contributions vasculaires aux troubles cognitifs (VCI) et à la démence faites par l'American Heart Association/ Académie américaine de neurologie18 (AHA/AAN) ont été également revues. Dans ce contexte, les recommandations de la 4e CCCDTD sont énoncées dans le Tableau 1.

Les points à retenir sont (1) une recommandation en faveur de l'adoption des critères diagnostiques des troubles cognitifs légers causés par la MA, à utiliser avec prudence et seulement

\section{Tableau 1 : Recommendations concernant les définitions de} la démence, de la MA et des troubles cognitifs vasculaires

* Nous conseillons l'adoption des critères de démence établis en 2011 par le groupe de travail INV/AA.

* Nous recommandons l'adoption des critères relatifs aux démences probable et possible causées par la MA présentés en 2011 par le groupe de travail INV/AA.

* Nous préconisons l'adoption des critères pour les troubles cognitifs légers provoqués par la MA soumis en 2011 par le groupe de travail INV/AA.

* Nous conseillons une réévaluation de l'utilité du concept de «MA prodromique »dans les années à venir lorsque les biomarqueurs seront disponibles, validés et prêts à l'emploi au Canada.

* Nous recommandons l'utilisation de la définition de « asymptomatique à risque de MA » donnée par le GIT seulement à des fins de recherche.

* étant donné que la présence de dépôts amyloïdes dans le cerveau chez les personnes normales est d'une signification indéterminée, nous déconseillons d'utiliser l'imagerie amyloïde chez des individus ne souffrant pas de perte de mémoire en dehors d'un contexte de recherche. Lors de discussions avec des patients, les médias ou des membres de la population, le milieu médical doit faire comprendre que la présence de dépôts amyloïdes dans le cerveau chez des personnes normales est d'une signification incertaine à l'heure actuelle.

* Nous approuvons les recommandations publiées en 2011 par les associations américaines AHA/AAN pour le diagnostic des troubles cognitifs d'origine vasculaire.
Tableau 2 : Recommendations concernant l'apparition d'une démence précoce

* Tous les patients se présentant avec une démence précoce doivent être aiguillés vers un centre de référence spécialisé en évaluation de la mémoire, de préférence doté d'un accès à des consultations et tests génétiques le cas échéant.

* Les dépenses pour les consultations et les tests génétiques doivent être prises en charge par l'état.

* Les médecins doivent être sensibilisés aux questions spécifiques relatives à l'apparition d'une démence précoce, notamment en ce qui concerne la perte d'emploi et l'accès aux services de soutien adaptés à cette tranche d'âge.

* étant donné la relative rareté de l'apparition d'une démence précoce, un registre national recensant les individus à risque, les porteurs de mutation et les patients présentant des symptômes facilitera la recherche dans le domaine thérapeutique.

* Ce registre doit être financé par l'état.

dans le cadre de pratiques cliniques spécialisées; (2) une forte recommandation de ne pas poser un diagnostic de «MA prodromique » en dehors d'un contexte de recherche; (3) les personnes ne présentant aucun symptôme de la MA doivent être classées comme «à risque de développer une MA» uniquement dans un contexte de recherche; (4) la mesure des dépôts amyloïdes dans le cerveau par le biais de l'imagerie TEP chez les personnes ne présentant aucun symptôme doit être effectuée uniquement dans un contexte de recherche.

Tableau 3 : Recommendations concernant la démence à progression rapide

* Une démence à progression rapide se définit comme une démence qui évolue significativement au cours des 12 mois suivants l'apparition des premiers symptômes cognitifs. $2 \mathrm{C}$

* Nous recommandons que les individus soupçonnés d'être atteints de démence à progression rapide soient référés à des médecins expérimentés qui ont accès aux ressources permettant une évaluation diagnostique systématique et complète. $2 \mathrm{C}$

* Après avoir exclu le delirium et les causes sous-jacentes évidentes de démence à progression rapide, nous suggérons d'adopter une stratégie diagnostique de cette maladie basée sur la prévalence des causes de ce type de démence à progression rapide suggérées par la littérature. 2B

* La stratégie diagnostique doit mettre l'accent sur la détection de maladies potentiellement traitables, telles que les infections, les maladies d'origine immunologique et celles liées à des anomalies métaboliques. 2B

* Chez les personnes souffrant de MA, en cas de détérioration de 3 points ou plus à l'échelle MMSE en 6 mois, ce qui identifie un groupe ayant un moins bon pronostic, il est conseillé d'analyser les facteurs de comorbidité et de réévaluer le traitement pharmacologique. 2B 


\section{Tableau 4 : Recommendations de la 2e CCCDTD [4] sur les indications de la tomodensitométrie radiologique}

- patient avec une démence âgé de moins de 60 ans

- détérioration rapide (c.-à-d. 1 ou 2 mois) et inexpliquée des facultés cognitives ou de l'état fonctionnel

- durée « courte » de la démence (moins de 2 ans)

- traumatisme crânien grave survenu récemment

- symptômes neurologiques inexpliqués (apparition de maux de tête violents ou de crises épileptiques graves par exemple)

- antécédents de cancer (en particulier les cancers qui font des métastases au cerveau)

- utilisation d'anticoagulants ou antécédents de troubles de la coagulation

- antécédents d'incontinence urinaire et de troubles de la marche dans les premiers stades de la démence (possibilité d'hydrocéphalie à pression normale)

- apparition d'anomalies focales (une hémiparésie ou un signe de Babinski par exemple)

- symptômes cognitifs ou tableau clinique inhabituels ou atypiques (aphasie progressive par exemple)

- troubles de la marche

\section{Apparition d'une démence précoce}

Dans le cadre d'un effort international visant à traiter les personnes souffrant de mutations provoquant l'apparition précoce de $\mathrm{MA}^{1}$ de type familial, et de demandes fréquentes de consultation en clinique de la mémoire pour des personnes d'âge moyen, le sujet des démences précoces, c'est dire apparaissant avant l'âge de 65 ans, a été revu. Les recommandations dans le Tableau 2 ont été approuvées par un consensus de $\geq 80 \%$.

Le message principal est le suivant : les patients atteints de démence avant l'âge de 65 ans doivent être référés à un spécialiste, de préférence dans une clinique où les consultations et les tests génétiques appropriés sont disponibles. En 1999, la CCCDTD a recommandé que tous ces patients soient dirigés vers un spécialiste. La CCCDTD actuelle conseille que ces

\section{Tableau 5 : Recommendations concernant la TEP 18F $\neg$ FDG et l'imagerie en tomographie d'émission monophotonique (TEM) du flot sanguin cérébral (FSC)}

* Pour les patients souffrant de démence, ayant subi une évaluation clinique standard et les tests d'imagerie de première ligne appropriés, et ayant été évalués par un médecin spécialiste des démences, mais pour lesquels le processus pathologique sous-jacent reste inconnu, empêchant un traitement clinique adéquat, nous recommandons la réalisation d'une TEP ${ }^{18} \mathrm{~F}-\mathrm{FDG}$ à des fins de diagnostic différentiel (1B).

* Si ces patients ne peuvent pas passer une TEP ${ }^{18} \mathrm{~F}-\mathrm{FDG}$, nous conseillons de procéder à une étude du FSC par imagerie TEM, à des fins de diagnostic différentiel (2C).
Tableau 6 : Recommendations concernant l'imagerie structurelle, tomodensitogramme et IRM

- Nous conseillons la réalisation d'une IRM cérébrale si un radiologue/neuroradiologue et/ou un spécialiste des troubles cognitifs (neurologue, gériatre ou géronto-psychiatre) est disponible pour interpréter un tel examen dans un contexte de démence, l'IRM pouvant alors s'avérer utile pour aider le spécialiste en charge du malade à établir le diagnostic et le pronostic liés à sa maladie (2B)

- Nous recommandons que des séquences standardisées d'acquisition des données IRM soient définies puis adoptées par les spécialistes en IRM oeuvrant dans les centres canadiens évaluant des malades avec démences, et soient utilisées en particulier lors d'évaluations répétées de ces malades. (2B)

- En plus des indications concernant l'imagerie structurelle énoncées précédemment, un tomodensitogramme ou une IRM doit être effectué dans le cadre de l'évaluation d'une personne atteinte de troubles cognitifs quand la découverte d'une maladie cérébrovasculaire insoupçonnée est susceptible de conduire à des modifications du traitement clinique.

- Nous recommandons, lorsque cela est possible que le spécialiste en charge des malades présente à ceux-ci les résultats des études d'imagerie qu'ils ont subi afin de les rendre plus conscients des changements se produisant dans leur cerveau, ce qui pourrait les motiver à adhérer à des programmes visant à modifier les facteurs de risque vasculaires qui peuvent aggraver leur état (3C)

Tableau 7 : Recommendations concernant I'IRM fonctionnelle

* Nous déconseillons le recours à l'IRM fonctionnelle dans le cadre de l'évaluation clinique des patients se plaignant de troubles cognitifs. (1B)

* Les études à venir devront définir des protocoles expérimentaux et des séquences standardisées d'acquisition afin de permettre la mise en commun des données. (1C; R)

* Il est présentement impossible sans de nouvelles études portant sur des nombres suffisants de malades, suivis de façon prolongée, de se prononcer sur la capacité de l'IRM fonctionnelle de détecter des changements associés aux stages précoces d'une démence, ou annonciateurs de la transformation de troubles cognitifs légers en MA. (1B; R)

* Les mêmes remarques s'appliquent à la possibilité d'utiliser l'IRM fonctionnelle pour distinguer la MA de démence non-MA, tels que la DFT et la DCL. (1B; R)

* Les mêmes remarques s'appliquent à la possibilité d'utiliser l'IRM fonctionnelle pour évaluer des changements de l'activité cérébrale en réponse à des interventions telles que l'entraînement cognitif et la pharmacothérapie. (1C; R)

* Les mêmes remarques s'appliquent à la possibilité d'utiliser l'IRM fonctionnelle pour mieux comprendre les mécanismes associés à des manifestations de type neuropsychiatrique survenant au cours d'une démence, ce qui pourrait conduire à de nouvelles approches thérapeutiques pour ces conditions. $(2 \mathrm{C}, \mathrm{R})$ 
Tableau 8 : Recommendations concernant l'imagerie TEP amylö̈de

* Bien que l'imagerie amyloïde constitue une technique prometteuse dans l'évaluation de la démence, bon nombre d'éléments pouvant avoir un impact sur son utilité diagnostique demeurent mal compris et nous recommandons donc que son usage soit réservé au domaine de la recherche pour le moment. $(1 \mathrm{C} ; \mathrm{R})$

* L'utilisation de l'imagerie amyloïde n'est pas approuvée au Canada à l'heure actuelle. Si toutefois l'imagerie amyloïde devait être mise à la disposition des cliniciens canadiens dans l'avenir, cette technique ne doit certainement pas être considérée être un test de première ligne et nous conseillons de la réserver pour l'évaluation approfondie de tableaux complexes et atypiques déjà suivis dans des cliniques de la mémoire fournissant des soins tertiaires, lorsqu'un diagnostic clinique plus précis est requis. (1B)

* Si toutefois cette technique devait être mise à la disposition des cliniciens canadiens dans l'avenir, nous déconseillons son usage pour des individus ne souffrant pas de déficience cognitive ou à des fins de d'évaluation initiale des personnes se plaignant de troubles cognitifs. (1B)

* Lorsqu'ils sont confrontés à des résultats d'examens de ce type réalisés dans d'autres pays, les médecins canadiens doivent être très prudents dans l'interprétation de ces résultats, et se rappeler qu'utilisé seul, cet examen ne peut pas diagnostiquer la MA, les troubles cognitifs légers, ou de distinguer un processus de vieillissement normal de celui associé à une démence. Nous préconisons qu'ils consultent un spécialiste des démences comprenant les limitations de cette technique.

* À l'heure actuelle, il n'existe aucune indication clinique pour l'imagerie amyloïde chez des individus ne souffrant pas de déficience cognitive, dans l'évaluation initiale des personnes se plaignant de troubles cognitifs, pour distinguer la MA d'autres types de démence également -associées à des dépôts d'amyloïde (comme la DCL et l'amylose cérébrovasculaire), pour différencier les unes des autres les variantes cliniques de la MA (la MA classique amnésique, l'ACP ou l'APP logopénique par exemple), ou encore différencier les uns des autres les sous-types moléculaires des DLFT.

* Dans les établissements de recherche proposant l'imagerie amyloïde, les chercheurs doivent être encouragés à élaborer des projets qui valideront l'usage de cette technique à des fins clinique et de recherche et qui évalueront la possibilité de l'appliquer en clinique (R).

* Les personnes planifiant ces projets sont fortement encouragées à employer cette technique pour (1) réduire l'hétérogénéité des groupes de personnes souffrant de troubles cognitifs légers recrutées pour des études cliniques; (2) identifier des cohortes de sujets qui répondront possiblement à un médicament possédant des propriétés anti-amyloïdes; et (3) étudier les patients susceptibles de développer une MA dans un délai relativement court $(\mathrm{R})$.

* L'utilisation de cette technique de façon longitudinale doit être réservée au domaine de la recherche pour les individus ne présentant aucun symptôme, les patients atteints de déficience cognitive subjective ne satisfaisant pas les critères de troubles cognitifs légers, ou les personnes à risque de développer une MA (porteurs de mutation génétique, sujets avec antécédents familiaux de MA, sujets porteurs d'ApoE $\varepsilon 4$ par exemple) (R).

* La recherche utilisant cette technique devra se pencher sur (1) l'évolution naturelle de la charge amyloïdienne et son rôle dans la physiopathologie de la MA et autres démences, (2) son utilisation en tant que potentiel marqueur d'efficacité de thérapies anti-amyloïdes, (3) la valeur des nouveaux traceurs amyloïdes marqués au ${ }^{18} \mathrm{~F}$, (4) la corrélation entre les études de pathologie tissulaire et les résultats TE de l'imagerie de l'amyloïde et (5) la corrélation entre les données de l'imagerie amyloïde et celles de mesure des biomarqueurs de MA dans le LCR ainsi que les marqueurs de dégénérescence neuronale (IRM structurale et imagerie métabolique en TEP FDG) (R).
Table 9 : Recommendations concernant la spectroscopie RM

- La spectroscopie en résonance magnétique s'annonce prometteuse puisqu'elle pourrait permettre de prédire quelles personnes souffrant de troubles cognitifs légers sont susceptibles d'évoluer vers une démence. Cependant, à l'heure actuelle, cette technique n'est pas recommandée pour un usage clinique de ce type chez les personnes présentant des troubles cognitifs légers. $(2 \mathrm{C} ; \mathrm{R})$

- La spectroscopie du proton $(\mathrm{H}+)$ demeure une technique prometteuse pour l'identification des sujets souffrant de troubles cognitifs légers dont la maladie va évoluer vers une démence. De nouvelles études longitudinales multi-sites doivent être menées afin d'établir des valeurs normatives. Ces études doivent suivre des critères standardisés d'éligibilité, de diagnostic et de méthodes d'acquisition des données, et comprendre une analyse automatisée du spectre incorporant une connaissance préalable de la forme de celui-ci pour divers métabolites (R).

- L'acquisition de données et les méthodes d'analyse normalisées doivent être conformes aux recommandations de l'International Society of Magnetic Resonance in Medicine (R).

- Les études de spectroscopie du proton visant à démontrer son efficacité clinique doivent être réalisées, lorsque cela est disponible, avec des systèmes d'IRM 3 Tesla afin d'améliorer la qualité des données $(\mathrm{R})$.

patients soient référés à des collègues possédant une expertise spécifique dans ce domaine.

\section{Démence à progression rapide}

On connait maintenant mieux les diverses causes de démences à progression rapide, en particulier la maladie de Creutzfeldt-Jakob, une maladie à déclaration obligatoire au Canada, et la CCCDTD a jugé nécessaire de redéfinir le contexte de ces maladies pour aider les cliniciens à mieux diriger les patients et leur famille vers les services appropriés. De plus, la détérioration rapide pouvant se produire lors de démences d'apparition tardive, telle que la MA, est si courante qu'elle requiert une recommandation. Les recommandations énoncées dans le Tableau 3 ont été approuvées à l'unanimité.

Les points à retenir sont les suivants : (1) les patients atteints de démence à progression rapide et pour lesquels le diagnostic demeure incertain, doivent être dirigés vers les services

\section{Tableau 10: Recommendations concernant d'autres méthodes de neuro-imagerie}

- L'emploi de biomarqueurs de neuroinflammation ou de pathologie tau en imagerie chez des patients souffrant de démence n'est pas recommandé à des fins cliniques.

- Bien qu'un nombre croissant d'ouvrages encouragent l'utilisation d'agents d'imagerie présynaptiques de la dopamine afin de distinguer la démence à corps de Lewy de la MA, ces agents d'imagerie ne sont pas recommandés pour une utilisation clinique. 
spécialisés appropriés, (2) les patients souffrant de MA qui manifestent une détérioration plus rapide que prévu doivent être réévalués pour éliminer des maladies associées.

\section{Neuroimagerie}

La neuro-imagerie a été le sujet le plus complexe abordé au cours de cette ronde de discussions CCCDTD. Tenant compte des progrès techniques accomplis dans ce domaine, les discussions ont conduit à des recommandations générales d'une part, et d'autre part portant plus spécifiquement sur la neuroimagerie structurelle (TDM et IRM), l'IRM fonctionnelle, l'imagerie TEP (discussions à la fois sur l'imagerie du ${ }^{18} \mathrm{~F}-\mathrm{FDG}$ et l'imagerie des ligands de l'amyloïde), la mesure du flot sanguin cérébral par tomographie d'émission monophotonique (TEM) et enfin la spectroscopie IRM (Tableaux 5-10). La plupart des recommandations sont destinées au domaine de la recherche, puisqu'à l'heure actuelle rares sont les tests disponibles universellement pour utilisation clinique. La question de savoir si tous les patients atteints de démence doivent subir ou non un examen par imagerie structurelle est discutée lors de chaque conférence CCCDTD, l'opinion générale étant que cet examen n'est pas nécessaire pour tous les patients, mais demeure utile pour ceux qui présentent des caractéristiques cliniques spécifiques (Tableau $4 \mathrm{du}^{2}$ ). Un des participants a suggéré que le critère d'âge (moins de 60 ans) dans la liste des critères de définition de l'utilité de ces études était purement arbitraire et devait donc être abandonné. Les participants ont voté contre la recommandation selon laquelle au moins un examen par imagerie structurelle devait être effectué afin de détecter la présence de maladie cérébrovasculaire insoupçonnée sur le plan clinique et d'exclure toute étiologie structurelle potentiellement réversible chez les personnes souffrant de troubles cognitifs (26 $\%$ d'appui seulement). La recommandation encourage Santé Canada à approuver l'utilisation de l'imagerie TEP des dépôts amyloïde dans des cliniques de soins tertiaires soignant la démence n'a pas atteint le consensus de consensus (63\% seulement l'ont appuyée). Un consensus partiel seulement a été obtenu pour la proposition suivante : chez les patients atteints de troubles cognitifs légers évalués par un spécialiste des démences et pour lesquels la gestion clinique serait influencée par la présence d'un processus neurodégénératif sous-jacent, une TEP ${ }^{18} \mathrm{~F}$-FDG doit être réalisée ou, si cette dernière n'est pas disponible, une TEM du FSC doit être effectuée (72\%).

Le message principal est que l'imagerie structurelle n'est pas nécessaire pour toutes les personnes souffrant de troubles cognitifs (quoiqu'indiquée pour la majorité). Bien qu'elle soit plus chère et moins accessible, l'IRM est préférable à la TDM. Le cas échéant, il est possible d'avoir recours à la TEP ${ }^{18}$ F-FDG et/ou à l'imagerie TEM du FSC à des fins cliniques sur des patients atteints de démences atypiques. L'imagerie des plaques amyloides, si elle devient accessible au Canada, pourrait être utile dans des cas spécifiques suivis par des spécialistes des démences.

\section{Biomarqueurs liquides}

L'examen des niveaux de protéines Aß42 et tau dans le liquide céphalorachidien (LCR) est un des biomarqueurs de la MA selon les critères du GIT et de l'INV/AA, et il a donc été considéré important d'évaluer la faisabilité et le bien-fondé d'un
Tableau 11: Recommendations concernant les biomarqueurs liquides

- Les niveaux dans le plasma A $\beta 1-42$ ne sont pas recommandés pour une utilisation clinique

Tableau 12: Recommendations concernant les traitements symptomatiques

* De nombreux cas de démence sont causés par plus d'une maladie. Généralement, il s'agit de la MA associée à une autre pathologie cérébrale. Dans ce cas, nous recommandons de baser la gestion de la maladie sur celui des diagnostics que l'on croit être la cause principale du tableau clinique. (1B)

* Nous conseillons l'utilisation des IC en tant qu'option de traitement de la MA accompagnée d'une maladie cérébrovasculaire. (1B)

* Nous préconisons l'utilisation des IC en tant qu'option de traitement de la démence associée à la maladie de Parkinson. (1A)

* Les données nécessaires pour décider de l'utilité des IC disponibles actuellement pour traiter la démence vasculaire sont limitées et contradictoires, et ne permettent pas de tirer de conclusion satisfaisante sur ce sujet.

* Les trois IC disponibles ont démontré leur efficacité à traiter la MA légère à sévère. Nous recommandons de traiter avec ces IC la majorité des patients atteints de MA (1A)

* Des comparaisons directes ne semblent pas indiquer de différence entre les IC. (2B) La décision d'utiliser un agent ou un autre doit s'appuyer sur le profil des effets indésirables, la facilité de manipulation, ainsi que sur les différences existant entre les agents dans leur pharmacocinétique et mécanismes d'action.

* Un traitement associant un IC à la mémantine est cohérent (puisque ces médicaments ont des mécanismes d'action différents) et semble sécuritaire, mais il n'y a pas de données permettant de conseiller ou déconseiller l'usage de cette combinaison. (2B)

* Dans le cas où le patient ne répond pas de façon adéquate aux interventions non pharmacologiques ou souffre de dépression ou dysthymie sévère ou de labilité émotionnelle, nous suggérons un essai avec un antidépresseur. (2A)

* Il y a suffisamment de données pour déconseiller l'utilisation du valproate en cas d'agitation et d'agressivité chez les personnes atteintes de MA. (1A)

* Les données sont insuffisantes pour émettre une opinion sur l'efficacité des IC et/ou de la mémantine dans le traitement des symptômes neuropsychiatriques. (2B)

* Nous recommandons l'utilisation de la rispéridone, de l'olanzapine et de l'aripiprazole en cas d'agitation, d'agressivité et de psychose associées à la démence, lorsque ces états représentent un risque pour la santé du patient et/ou des autres personnes. Les avantages potentiels des antipsychotiques doivent être évalués en fonction des risques réels de complications liées à leur utilisation, telles que les accidents vasculaires cérébraux et la mort (2A)

* Les données sont insuffisantes pour émettre une opinion sur l'efficacité de la quétiapine dans le traitement de l'agitation sévère, de l'agressivité et de la psychose associées à la démence. (2B)

* Les données sont insuffisantes pour émettre une opinion sur l'efficacité des ISRSs ou de la trazodone dans le traitement des patients déments agités. (2B) 
Tableau 13: Recommendations concernant l'interruption des inhibiteurs de la cholinestérase

* Arrêter les IC chez des patients souffrant d'une MA modérée à sévère peut aggraver le deficit cognitif et fonctionnel (2B). Le risque d'effets secondaires et le prix des médicaments font partie des éléments à considérer dans le cas où la thérapie se poursuit. Il est recommandé d'interrompre les inhibiteurs de la cholinestérase lorsque :

a) Le patient et/ou son mandataire décident d'arrêter le traitement après avoir été informé des risques et des avantages de poursuivre ou d'arrêter les IC;

b) Le patient ne respecte pas la posologie choisie et continuer le traitement serait inutile, et il est impossible de mettre en place un système pour rectifier le problème;

c) L'état clinique du patient s'est aggravé suite à l'instauration du traitement;

d) Le patient éprouve des effets secondaires intolérables qui sont certainement ou probablement dus aux IC;

e) Les autres maladies dont souffre le patient rendent le risque associé à l'utilisation du traitement inacceptable, ou alors sont si sévères que le traitement est futile (patient en phase terminale par exemple);

f) La démence du patient a progressé pour arriver à un stade (stade 7 sur l'échelle de détérioration globale par exemple) où aucun avantage significatif sur le plan clinique ne découle du traitement.

* Lorsque la décision d'arrêter le traitement a été prise en raison d'un manque d'efficacité perçu, nous recommandons la réduction des doses avant l'interruption de l'agent ainsi qu'une surveillance étroite du patient durant les 3 premiers mois afin de déceler une détérioration nouvelle. Si une telle détérioration se produit, il faut considérer la reprise du traitement. (2C)

examen du LCR à des fins de diagnostic général ou pour des cas atypiques. Tous les participants ont convenu que la mesure des concentrations plasmatiques du peptide $\mathrm{A} \beta 42$ n'est pas utile et qu'elle n'est donc pas recommandée pour une utilisation clinique (Tableau 11). La recommandation de mesurer les concentrations de protéines $A ß 42$ et tau dans le liquide céphalorachidien (LCR) n'a pas été retenue (64\%), ni celle suggérant que les mesures de protéines Aß42, de tau et de tau phosphorylée en Ser 181 dans le LCR soient réalisées uniquement par des laboratoires possédant une technologie validée et participant activement à des programmes de contrôle de la qualité $(71 \%)$.

Le message à retenir est que pour l'instant, la mesure des concentrations de protéines Aß42 et tau dans le LCR n'est pas pertinente d'un point de vue clinique, bien que ces techniques fassent partie des protocoles de recherche dans les études thérapeutiques et d'observation.

\section{Mise à jour sur les traitements symptomatiques}

Bien qu'aucun nouveau médicament n'ait été approuvé au Canada ou dans d'autres pays depuis la 3e conférence CCCDTD de 2006, nous avons considéré comme important d'examiner les nouvelles données concernant les indications et la meilleure façon d'utiliser ces médicaments (Tableau 12). L'accent a été mis sur les règles d'interruption des IC qui n'avaient pas été clairement définies dans la littérature auparavant (Tableau 13).

Les messages importants sont que (1) les causes sous-jacentes à la démence doivent être traitées; (2) les IC sont recommandés pour la MA aux stades de démence légère à sévère, de MA associée à une maladie cérébrovasculaire, de démence liée à la maladie de Parkinson, mais pas dans le cas d'une démence probablement vasculaire; (3) l'association des IC à la mémantine est logique, mais aucun avantage additif n'a été démontré de façon concluante; (4) pour les cas d'agitation sévère, les antipsychotiques atypique tels que la rispéridone, l'olanzapine et l'aripiprazole sont conseillés, toutefois les risques de cette thérapie doivent être pris en considération; (5) les données concernant les IC, la mémantine, les ISRSs ou la trazodone en tant que thérapie de première ligne visant à traiter les symptômes neuropsychiatriques sont insuffisantes; (6) le valproate ne doit pas être utilisé en cas d'agitation ou de comportement agressif.

\section{Conclusions}

La 4eCCCDTD a conclu que malgré des progrès importants, des changements fondamentaux pour le diagnostic et la gestion des démences se font toujours attendre. Les critères du GIT et de l'INV/AA servent en grande partie à codifier les pratiques des établissements spécialisés dans le traitement de la démence et des troubles cognitifs légers découlant de la MA et dans la recherche à tous les stades de la MA. En conséquence, les médecins canadiens qui ne sont pas experts en démence ne seront que peu affectés par les recommandations de la 4e CCCDTD.

La conférence de 1999 avait recommandé que les patients plus jeunes ( $<$ de 65 ans) et les patients souffrant de démence à progression rapide soient référés vers des spécialistes de la démence, ce qui est toujours le cas aujourd'hui, avec cependant en plus une recommandation de diriger ces malades non seulement vers des spécialistes des démences, mais vers des spécialistes s'intéressant spécifiquement à de tels patients.

Si à l'avenir le recours aux biomarqueurs pour le diagnostic des démences s'avère justifié par des études de bonne qualité, cela modifiera la façon dont le déclin cognitif est évalué et aura probablement des répercussions économiques très importantes. Dès maintenant, les médecins canadiens menant des recherches sur les démences doivent considérer la façon dont les nouveaux critères de recherche influenceront la demande d'accès aux méthodes d'imagerie et aux tests réalisés en laboratoire, qui ne sont pas encore monnaie courante au Canada pour évaluer les personnes atteintes de démence.

\section{REMERCIEMENTS}

Nous souhaitons remercier sincèrement le personnel de Medplan qui nous a aidés à préparer les nombreuses téléconférences précédant la réunion et les systèmes de logistique utilisés lors de la 4e conférence CCDTD, ainsi que le personnel du Centre McGill d'études sur le vieillissement qui nous a aidés durant la conférence organisée à Montréal. 


\section{RÉFÉRENCES}

1. Organizing Committee Canadian Consensus Conference on the Assessment of Dementia. Assessing Dementia the Canadian Consensus. CMAJ. 1991;144(7):851-3.

2. Patterson $\mathrm{C}$, Gauthier $\mathrm{S}$, Bergman $\mathrm{H}$, et al. The recognition, assessment and management of dementing disorders: conclusions from the Canadian Consensus Conference on Dementia. CMAJ. 1999;160 Suppl:S1-15.

3. Chertkow H. Diagnosis and treatment of dementia: introduction. CMAJ. 2008;178(3):316-21.

4. Patterson C, Feightner JW, Garcia A, et al. Diagnosis and treatment of dementia: 1. Risk assessment and primary prevention of Alzheimer disease. CMAJ. 2008;178:548-56.

5. Chertkow H, Massoud F, Nasreddine Z, et al. Diagnosis and treatment of dementia: 3. Mild cognitive impairment and cognitive impairment without dementia. CMAJ. 2008;178: 1273-85.

6. Hogan DB Bailey P, Black S, et al. Diagnosis and treatment of dementia: 4. Approach to management of mild to moderate dementia. CMAJ. 2008;179:787-93.

7. Hogan DB, Bailey P, Black S, et al. Diagnosis and treatment of dementia: 5. Non-pharmacologic and pharmacologic therapy for mild to moderate dementia. CMAJ. 2008;179:1019-26.

8. Herrmann N, Gauthier S. Diagnosis and treatment of dementia: 6 . Management of severe Alzheimer disease. CMAJ. 2008;179: 1279-87.

9. Feldman HH, Jacova C, Robillard A, et al. Diagnosis and treatment of dementia: 2. Diagnosis. CMAJ. 2008;178:825-36.

10. Dubois B, Feldman HH, Jacova C, et al. Research criteria for the diagnosis of Alzheimer's disease: revising the NINCDSADRDA criteria. Lancet Neurol. 2007;6:734-46.

11. Dubois B, Feldman HH, Jacova C, et al. Revising the definition of Alzheimer's disease: a new lexicon. Lancet Neurol. 2010;9: 1118-27.
12. McKhann GM, Knopman DS, Chertkow H, et al. The diagnosis of dementia due to Alzheimer's disease: recommendations from the National Institute on Aging - Alzheimer's Association workgroups on diagnostic guidelines for Alzheimer's disease. Alzheimers Dement. 2011;7:263-9.

13. Albert MS, DeKosky ST, Dickson D, et al. The diagnosis of mild cognitive impairment due to Alzheimer's disease: recommendations from the National Institute on Aging Alzheimer's Association workgroups on diagnostic guidelines for Alzheimer's disease. Alzheimers Dement. 2011;7:270-9.

14. Sperling RA, Aisen P, Beckett L, et al. Toward defining the preclinical stages of Alzhheimer's disease: recommendations from the National Institute on Aging - Alzheimer's Association workgroups on diagnostic guidelines for Alzheimer's disease. Alzheimers Dement. 2011; 7: 280-92.

15. Gauthier S, Patterson C, Gordon M, Soucy JP, Schubert F, Leuzy A. Commentary on "Recommendations from the National Institute on Aging - Alzheimer's Association workgroups on diagnostic guidelines for Alzheimer's disease". Alzheimers Dement. 2011; 7:330-3.

16. The AGREE Collaboration. Development and validation of an international appraisal instrument for assessing the quality of clinical practice guidelines: the AGREE project. Qual Saf Health Care. 2003;12:18-23.

17. Guyatt GH, Oxman AD, Vist GE, et al for the GRADE Working Group. GRADE; an emerging consensus on rating quality of evidence and strength of recommendations. BMJ. 2008;336:9246.

18. Gorelick PB, Scuteri A, Black SE, et al. Vascular contributions to cognitive impairment and dementia: a statement for health care professionals from the American Heart Association/American Academy of Neurology. Stroke. 2011;42:2672-713.

19. Wu L, Rosa-Neto P, Hsiung GY, et al. Early-onset familial Alzheimer's disease (EOFAD). Can J Neurol Sci. 2012;39: $436-45$ 